\title{
Editorial: On IRIE Vol. 17
}

Do you have secrets? We do! We couldn't edit this journal without! Without the anonymous peer reviewing process e.g. or the well kept secrets of our finding the subject, the guest editors etc.. This way we can do our job efficiently and you can consume the outcome - efficiently.

Try to think of it the other way round: what if everything would be fully transparent, democratic and participatory. You would be involved in a complex, very interactive process that takes its time and demands costly commitment. You would have all the information but also all the obligations associated with them. Are you as a reader - willing to invest this effort or do you - for convenience reasons e.g. - accept this informative asymmetry. Informative asymmetries, that is what secrets finally are. And in many cases they are vital for the everyday functioning of so many procedures within our society. There are laws that protect these asymmetries because otherwise companies would go bankrupt, customers would be charged suboptimal prices and markets would collapse. Breaking these asymmetries and taking advantage of that is called insider trading, industrial espionage etc. and prosecuted by law.

On the other hand informative asymmetries are what originally markets are designed for to avoid. In a perfect market informed customers take informed decisions thus forcing companies to offer optimal prices (at least better prices than their competitors) for thus fully comparable offerings. Markets are designed to establish informative symmetries by maintaining and protecting informative asymmetries as stated above.

But not only economic structures are at stake. Take something more personal: the person itself - the meaning of the original latin notion 'persona' is 'mask'. It hides the face of the actor from the audience and is thus constitutive for the play. It hides the actor and presents the figure. Is this informative asymmetry associated with the notion 'persona' also constitutive for our being a 'person' - to keep some things hidden from others and present something defined to them? Rather to be a secret than to have secrets?

This special issue will explore the complex nature of "secrecy" in our contemporary information society. The ethical exploration of secrecy must be renewed in the face of the multiple and shifting social, political and cultural contexts in which information flows. And maybe this issue thus reduces some informative asymmetries only made possible by maintaining others as stated above.

We do thank the editors and authors of this issue for their admirable efforts to clarify the subject and questions concerned and look forward to your valuable feedback.

Sincerely yours,

The editors. 